\title{
Hybrid salivary gland tumor of the upper lip or just an adenoid cystic carcinoma? Case report
}

\author{
Adalberto Mosqueda-Taylor ${ }^{1}$, Ana Ma. Cano-Valdez ${ }^{2}$, José-Daniel-Salvador Ruiz-Gonzalez ${ }^{3}$, Cesar Ortega- \\ Gutierrez $^{4}$, Kuauhyama Luna-Ortiz ${ }^{4}$
}

${ }^{1}$ DDS. Departamento de Atención a la Salud, Universidad Autonoma Metropolitana Xochimilco

${ }^{2}$ MD. Departamento de Patología, Instituto Nacional de Cancerología

${ }^{3}$ MD. Neurosurgeon, Departamento de Cabeza y Cuello. Instituto Nacional de Cancerología

${ }^{4}$ MD. Departamento de Cabeza y Cuello, Instituto Nacional de Cancerología, México D.F.

Correspondence:

Departamento de Cabeza y Cuello

Instituto Nacional de Cancerología

Av. San Fernando 22, Col. Sección XVI,

Tlalpan, Mexico D.F. 14090 ,

kuauhyama@starmedia.com

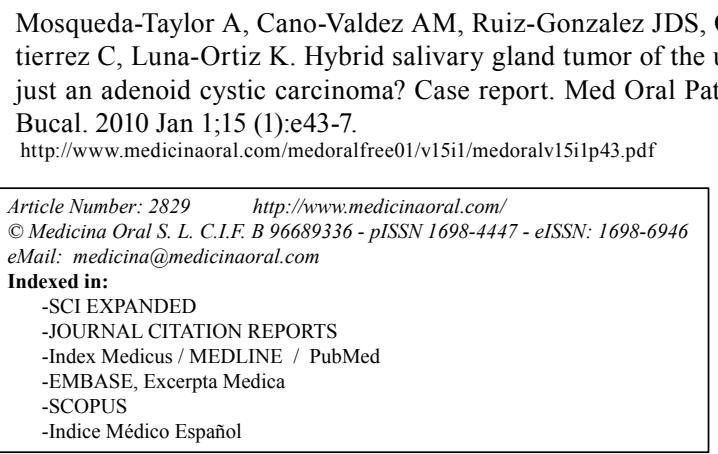

\begin{abstract}
A 65 year-old male patient with a one year-duration tumoral growth located in the upper lip was diagnosed on incisional biopsy as epithelial-myoepithelial carcinoma. After wide surgical excision the histopathological analysis revealed the lesion was composed predominantly $(>90 \%)$ of adenoid cystic carcinoma. In new sections it was found a very small and isolated area of adenoid cystic carcinoma at the bottom of the incisional biopsy. As surgical margins were free of lesion, no adjuvant treatment was given. The occurrence of a transitory ischaemic attack at 36 months of follow-up led to a neurological and MRI evaluation, which disclosed a well-defined $3.5 \times 3 \mathrm{~cm}$ lesion suggestive of metastasis, located on the right temporal area. The lesion was surgically removed and a histopathological diagnosis of neurocysticercosis was rendered. After 40 months of follow-up there is no evidence of recurrence. Conclusion: True hybrid tumors of salivary glands are rare and treatment in each case should be done according to the component with the higher aggressiveness. However, the occurrence of epithelial-myoepithelial carcinoma areas within an adenoid cystic carcinoma seems to be a frequent finding, and because both lesions share a common origin, some authors consider that this may not be a true hybrid neoplasm but a variant of the latter.
\end{abstract}

Key words: Carcinoma, hybrid tumor, adenoid cystic, upper lip. 


\section{Introduction}

Hybrid tumors of the salivary glands are lesions composed by two different neoplasms, each of which conforms with an exactly defined tumor category, arise in a single anatomical location, and eventually produce a unique clinical mass both clinically and microscopically (1). These are very rare lesions, as they represent less than $0.1 \%$ of all salivary gland neoplasms. To date, there are less than 30 cases reported in the literature, most of which have been detected in parotid and palatine glands $(2,3)$.

The purpose of this article is to present a case of hybrid carcinoma of upper lip salivary glands, composed by adenoid cystic carcinoma (ACC) and epithelial-myoepithelial carcinoma (EMC), as well as to discuss its probable histogenesis and to describe its salient clinico-patological features, diagnosis and therapeutic approach.

\section{Case Report}

A 65 year-old male patient consulted his physician because of a one year-duration tumoral growth located in the upper lip, which produced occasional pain on palpation. Apart from a daily 15-cigarette smoking history and moderate alcohol consumption during the last 40 years, his past medical history was unremarkable. Intraoral physical examination revealed a multilobulated, exophytic and ulcerated tumoral growth that measured $3 \times 2.5 \mathrm{~cm}$, located in the upper lip mucosa (Fig. 1). Incisional biopsy was diagnosed as EMC (Fig. 2), and immunohistochemical analysis was consistent with this diagnosis, as markers for cytokeratin were positive for inner ductal cells and smooth muscle actin was positive for the outer clear cells. The patient was treated by means of wide surgical excision, which included part of the orbicular muscle surrounding the lesional area. The surgical defect was covered with a skin flap and the patient was discharged on the next day without complications. Histopathologic study of the surgical specimen disclosed a neoplasm composed predominantly (more than $90 \%$ ) by sheets and cords of basaloid appearing hyperchromatic cells, with scant cytoplasm and ill defined cellular borders, which formed solid and cribriform multicystic structures filled by basophilic amorphous material and surrounded in some areas by eosinophilic material of basal lamina appearance, rendering a diagnosis of ACC (Fig. 3). Surgical margins were free of neoplasm. Aspiration cytology of the excided tumoral mass disclosed abundant viscous and granular material, and cytological smears were subsequently fixed on $96^{\circ}$ ethylic alcohol and then stained with H\&E. Small, uniform, cells with scanty cytoplasm were identified. Nuclei were round to oval with coarse chromatin and indistinct nucleoli, and the cells were scattered in ramified, cribriform and globoid architectural patterns. In the latter, cells were disposed surrounding eosinophilic

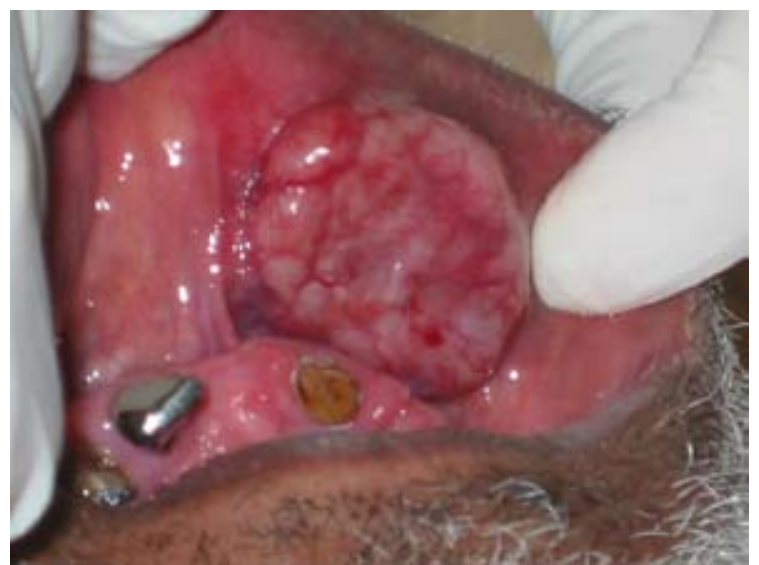

Fig. 1. Exophytic tumoral growth on the upper lip mucosa.

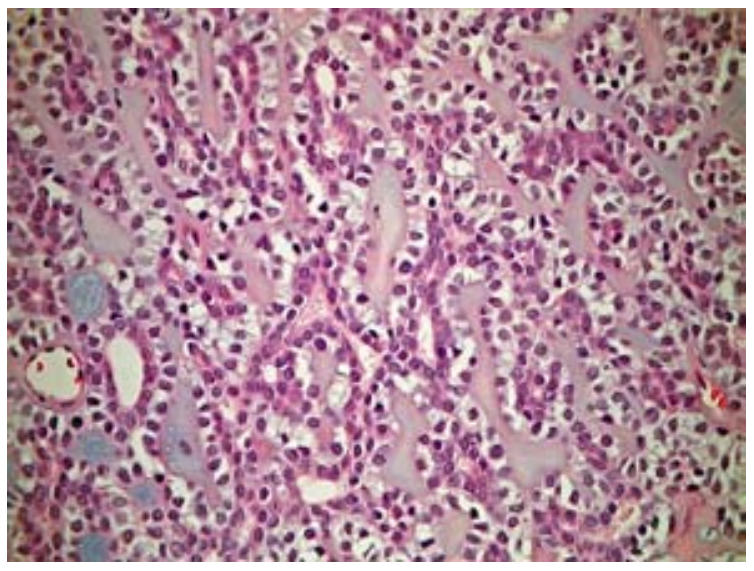

Fig. 2. Sheets of epithelial-myoepithelial carcinoma on incisional biopsy (H\&E x400).

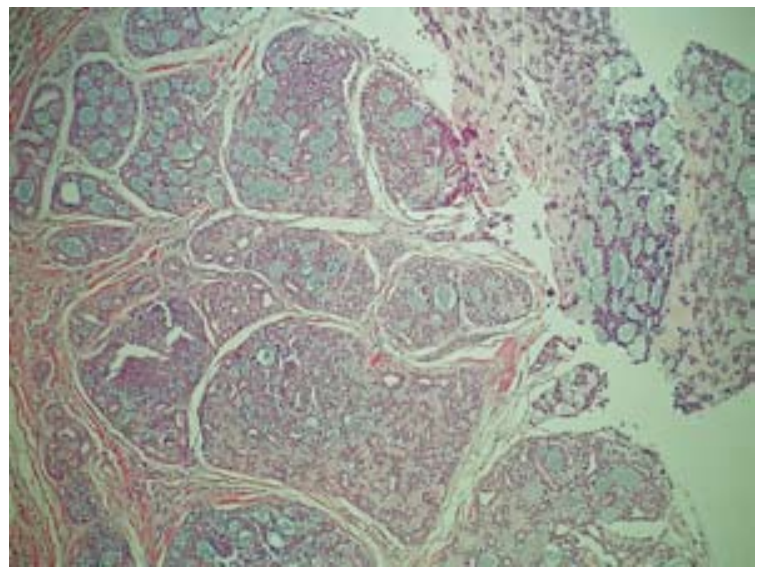

Fig. 3. Cribriform and solid areas of adenoid cystic carcinoma in excised specimen (H\&E x 100). 


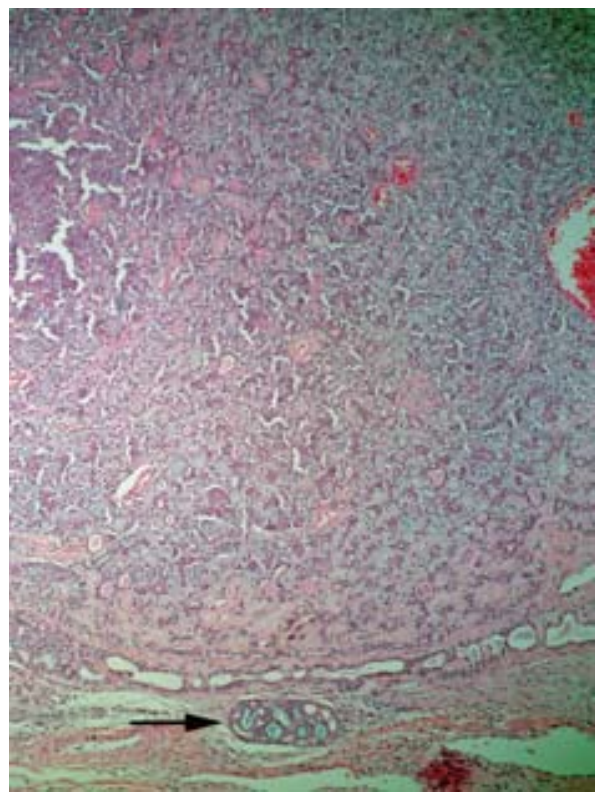

Fig. 4. Localized small foci of adenoid cystic carcinoma at the bottom of large sheets of epithelialmyoepithelial carcinoma in incisional biopsy (H\&E $\mathrm{x} 40$ ).

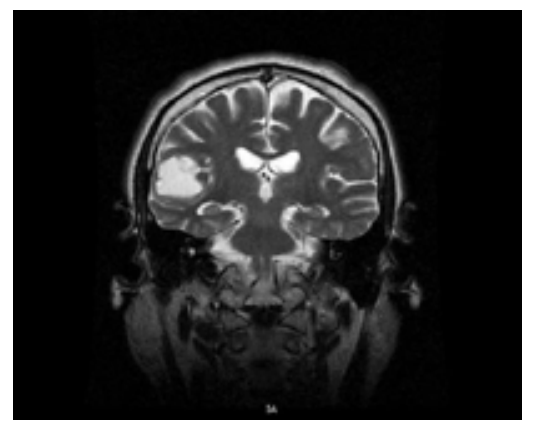

Fig. 5. MRI showing a lesion located in the right temporal area which was confirmed histopathologically as neurocysticercosis.

hyaline basal membrane-like material, which supported the diagnosis of ACC. On review of serial sections of the original incisional biopsy it was found a localized small foci of ACC at the base of the EMC, which confirmed the diagnosis of hybrid carcinoma of salivary gland origin in this case (Fig. 4). After three years the patient developed symptomatology suggestive of transitory ischaemic attack and neurological evaluation was performed. MRI disclosed a $3.5 \times 3 \mathrm{~cm}$ lesion located in the right temporal area (Fig. 5). With a presumptive diagnosis of metastasis he was sent to the operating room where a well-defined cystic lesion was surgically removed and the patient was released after 5 days with no post-operatory complications. Microscopic examination rendered a diagnosis of neurocysticercosis. Patient is alive with no evidence of disease after 40 months of follow-up.

\section{Discussion}

In 1996 Seifert and Donath introduced the term "hybrid tumor of salivary glands" to describe "very rare tumor entities which are composed of two different tumor entities, each of which conforms with an exactly defined tumor category. The tumor entities in a hybrid tumor are not separated but have an identical origin within the same topographical area" (1). This concept of hybrid tumor should not be confused with certain entities of the salivary glands that may exhibit two or more different morphologies in a single lesion, such as collision tumors (which are lesions that originate in separate regions but coalesce in a particular area), tumors with biphasic differentiation (single entities containing two different cellular types, such as pleomorphic adenomas), synchronous and multiple tumors, carcinomas with metaplastic change and other examples (2-4).

While the possibility of developing multiple tumors that coalesce and invade each other and the production of collision tumors that simulate a single entity does exist in major salivary glands, the probability of this phenomenon to occur in the minor salivary glands is remote, and therefore the diagnosis of a hybrid tumor and tumors with biphasic differentiation should be strongly considered when the tumor contains two different tumor entities which are not separated and are located in the same topographical area.

Since its original description, less than 30 cases of hybrid tumors of salivary gland origin have been reported to date (1-5). Most cases have developed within parotid and palatine glands (3), but this entity has been found also in submandibular glands $(2,5)$, maxillary sinus (4) and lacrimal glands (2). Although there are hybrid tumors with benign components, most of the reported cases are malignant neoplasms (hybrid carcinomas) in which the most frequently identified neoplasm has been ACC (14 out of 25 reported cases). The combination of ACC with EMC has been informed in seven instances, including the present case $(1,3,4,6)$; however, according to our review of the literature, our case is the first hybrid carcinoma reported to occur in the upper lip.

In 1972 Donath et al (7) introduced the term EMC, which has been also known as adenomyoepithelioma, clear cell carcinoma, glycogen-rich carcinoma and other synonyms. It comprises approximately $1 \%$ of all salivary gland neoplasms and most cases are diagnosed during the sixth and seventh decades of life, with a female predominance (F:M =2:1). Most cases are found in the major salivary glands, but it may also occur in minor glands of the oral mucosa and the upper and lower respiratory tract. Margin status is a major pathological prognostic factor, as incomplete surgical excision is associated with recurrence and metastases. Recurrences have occurred in around $40 \%$ of the cases and metastases in $14 \%(8)$. 
ACC comprise approximately $10 \%$ of all epithelial salivary gland neoplasms, and around $30 \%$ of epithelial minor salivary gland tumors. It occurs in all age groups with a higher frequency in middle-aged and older patients, with no apparent sex predilection. Factors that influence survival include histologic patterns, tumor site, clinical stage, bone involvement and status of surgical margins, and the estimated 5-year survival rate is approximately $35 \%$, decreasing to $10 \%-20 \%$ after 10 years. Wide surgical excision with and without post-operative radiation is still the treatment of choice $(9,10)$. Of the seven cases of hybrid carcinomas composed of $\mathrm{ACC}$ and EMC reported to date (including the present case), three have occurred in the palate, two in parotid, one in maxillary sinus and one in the upper lip. The case affecting the maxillary sinus occurred in the youngest patient (a 26 year-old woman) and behaved very aggressively. It recurred as ACC seven years after combined treatment with surgery and radiotherapy, invaded the intracranial space, produced metastases to multiple bones and caused the patient death (4). Age was known in five of the remaining six patients, and ranged from 62 to 71 years (mean $67 \mathrm{yr}$ ). Three patients were alive with no evidence of disease after 16, 20 and 49 months of follow up and the case in the series of Croitoru et al (6) was reported as alive without evidence of disease at the time of publication of their article, but the authors did not state the length of the follow-up. There is not information about the outcome of the other two cases.

Grenko et al (11) considered that the frequent occurrence of areas of ACC and EMC in the same neoplasm is because both lesions share common phenotypic histodifferentiation pathways, and these authors do not consider them as examples of hybrid carcinomas; instead, they suggest that when adenoid cystic carcinoma-like areas are seen in EMC these should be recognized as an anomalous differentiation that does not imply the synchronous development of two tumors, i.e., a hybrid neoplasm. Although they found three cases of ACC and two EMC with foci of the other neoplasm and confirmed that both components had immunohistochemical and ultrastructural similarities, the treatment in each case was performed according to the predominant neoplasm. The problem in some cases, as occurred in the present one, is that the predominant (and perhaps the unique) tissue found in the initial biopsy could be the smallest and less aggressive tumoral component found in the surgical specimen, a situation that may lead to perform a more conservative and perhaps inadequate treatment that favors the persistence or recurrence of the more aggressive neoplasm. In the present case surgical management was performed under oncological considerations after a diagnosis of malignancy, which allowed the entire removal of the lesion with wide surgical margins. As the probability of cervical lymph node metastasis in EMC is relatively low, we decided not to perform neck dissection in this case, as such procedure has to be considered therapeutic and not an elective treatment for this type of neoplasm.

Some authors consider that completely divergent differentiation in a single tumor may lead to the appearance of two well-defined neoplasms or a true hybrid tumor $(1,2,6)$. An important issue is that the prognosis of an hybrid carcinoma is not dependent on a certain amount of each of the two neoplastic categories present in each case, as it has been demonstrated that there are some cases in which the more aggressive component represent scarcely $20 \%$ of the tumor and it had produced metastasis (6). Therefore, treatment in each case should be performed according to the more aggressive component, which is the therapeutic recommendation in all cases of hybrid tumors of salivary gland origin (1-4,6), and in all cases a close and long follow-up is necessary in order to determine if the biologic behavior of these lesions is similar to that of the individual neoplasms that compose such hybrid tumors.

Although the existence of true hybrid tumors is well established, the fact that most of the reported cases of hybrid tumors of salivary gland origin are composed by ACC and EMC led some authors to consider the possibility that these are in fact a biphasic differentiation expressed by ACC and therefore careful examination of surgical specimens reported as EMC should be done in order to exclude the presence of typical areas of ACC, which should determine treatment and prognosis.

\section{References}

1. Seifert G, Donath K. Hybrid tumours of salivary glands. Definition and classification of five rare cases. Eur J Cancer B Oral Oncol. 1996;32B:251-9.

2. Nagao T, Sugano I, Ishida Y, Asoh A, Munakata S, Yamazaki K, et al. Hybrid carcinomas of the salivary glands: report of nine cases with a clinicopathologic, immunohistochemical, and p53 gene alteration analysis. Mod Pathol. 2002;15:724-33.

3. Ruíz-Godoy LM, Mosqueda-Taylor A, Suárez-Roa L, Poitevin A, Bandala-Sánchez E, Meneses-García A. Hybrid tumours of the salivary glands. A report of two cases involving the palate and a review of the literature. Eur Arch Otorhinolaryngol. 2003;260:312-5.

4. Woo JS, Kwon SY, Jung KY, Kim I. A hybrid carcinoma of epithelial-myoepithelial carcinoma and adenoid cystic carcinoma in maxillary sinus. J Korean Med Sci. 2004;19:462-5.

5. Snyder ML, Paulino AF. Hybrid carcinoma of the salivary gland: salivary duct adenocarcinoma adenoid cystic carcinoma. Histopathology. 1999;35:380-3.

6. Croitoru CM, Suarez PA, Luna MA. Hybrid carcinomas of salivary glands. Report of 4 cases and review of the literature. Arch Pathol Lab Med. 1999;123:698-702.

7. Donath K, Seifert G, Schmitz R. Diagnosis and ultrastructure of the tubular carcinoma of salivary gland ducts. Epithelial-myoepithelial carcinoma of the intercalated ducts. Virchows Arch A Pathol Pathol Anat. 1972;356:16-31.

8. Fonseca I, Soares J. Epithelial-myoepithelial carcinoma. In: Barnes L, Eveson JW, Reichart PA, Sidransky D. (Eds). Pathology and Genetics Head and Neck Tumours. World Health Organization Classification of Tumours. Lyon: IARC Press. 2005. p. 225-6.

9. El-Naggar AK, Huvos AG. Adenoid cystic carcinoma. In: Barnes 
L, Eveson JW, Reichart PA, Sidransky D. (Eds). Pathology and Genetics Head and Neck Tumours. World Health Organization Classification of Tumours. Lyon: IARC Press. 2005. p. 221-2.

10. Luna Ortiz K, Carmona Luna T, Herrera Gómez A, Cano Valdez AM. Macroglossia caused by adenoid cystic carcinoma. Case report. Med Oral Patol Oral Cir Bucal. 2008;13:E395-7.

11. Grenko RT, Abendroth CS, Davis AT, Levin RJ, Dardick I. Hybrid tumors or salivary gland tumors sharing common differentiation pathways? Reexamining adenoid cystic and epithelial-myoepithelial carcinomas. Oral Surg Oral Med Oral Pathol Oral Radiol Endod. 1998;86:188-95. 